\title{
Adjuvant TKIs: still an optimal choice
}

\author{
Si-Yang Liu, Yi-Long Wu \\ Guangdong Lung Cancer Institute, Guangdong General Hospital \& Guangdong Academy of Medical Sciences, Guangzhou 510080, China \\ Correspondence to: Yi-Long Wu. Guangdong Lung Cancer Institute, Guangdong General Hospital \& Guangdong Academy of Medical Sciences, \\ Guangzhou 510080, China. Email: syylwu@live.cn. \\ Provenance: This is an invited article commissioned by the Section Editor Mong-Wei Lin (Division of Thoracic Surgery, Department of Surgery, \\ National Taiwan University Hospital and Taiwan University College of Medicine, Taipei). \\ Response to: Ye T, Chen H. Adjuvant targeted therapy for resected NSCLC: to be or not to be? J Thorac Dis 2018;10:S3297-9.
}

Submitted Sep 24, 2018. Accepted for publication Nov 12, 2018.

doi: $10.21037 /$ jtd.2018.11.67

View this article at: http://dx.doi.org/10.21037/jtd.2018.11.67

Thanks for Pro. Hai-Quan Chen and colleagues for their interest in our study and appreciate it raises questions about the trial design and eventual OS benefits for EGFR mutant patients received adjuvant TKI therapy (1).

During the last decade, targeted therapy has yielded encouraging results with substantial progress for molecular subgroups of patients with advanced disease. Lung cancer treatment has stepped into precise medicine era along with the accumulation of driver mutations knowledge through emerging technology platforms (e.g., next-generation sequencing) and the development of new drugs that specifically target molecular abnormalities (2). Since IPASS study published ten years ago, the superiority of EGFR TKIs in survival and safety benefits has established its firstline treatment status in advanced EGFR mutated NSCLC and completely replaced chemotherapy.

While for completely resected stage II-IIIA NSCLC, platinum-based doublet therapy has always been the standard of care. However patients received adjuvant chemotherapy could only get a 4-5\% improvement in 5 -year overall survival (OS) compared to surgery alone and have to suffer adverse events at the same time (3). While EGFR-TKIs have achieved this miracle success in advanced NSCLC, what about its application in adjuvant settlement?

Nevertheless forepassed studies were not designed specifically for patients with $E G F R$ mutations so that adjuvant TKIs have not shown meaningful effects (4).

Given the facts above, we conducted this phase III clinical trial in EGFR-mutant patients to see if adjuvant gefitinib could be an optimal choice.

After determining the research objectives, study design was the primary problem we had to face. First of all, in addition to EGFR mutations, enrolled patients were set up as stage II-IIIA with N1 or N2 lymph nodes metastasis since this specialized subset would achieve the more benefit from adjuvant chemotherapy. Previous studies have shown that the median disease free survival (DFS) for stage II and III was from 9.0 to 21.0 months, as a result the duration of EGFR-TKIs was set up as 24 months in order to reduce recurrence. Secondly patients in the experimental arm in our study were arranged to receive TKIs right after surgery without chemotherapy at first. When we had a look back to previous studies, such as RADIANT, negative results were finally obtained when chemotherapy was given at first (5). So here we meant to compare adjuvant EGFR-TKIs with chemotherapy directly. At last let's turn our attention to the setting of study endpoints. In this study DFS and OS was set up as the primary and secondary endpoints respectively. This was a prudent decision according to the findings by Mauguen and colleagues that disease-free survival is an appropriate surrogate endpoint for OS (6). Furthermore although OS benefit has been considered as a crucial indication for changing the clinical practice, it is worth notice that in our phrase III research patients received chemotherapy in the control arm other than placebo. So even if OS in EGFR-TKIs group was the same as chemotherapy eventually, the superiority of DFS data and safety profile of adjuvant EGFR-TKIs would still be an optimal choice for elder patients. In recently published EVAN study, adjuvant erlotinib also obtained significant survival benefits (7). We are relieved to see these two results can be mutually corroborated. 
Table 1 (Neo)adjuvant clinical trials conducted in Guangdong Lung Cancer Institute

\begin{tabular}{lcccc}
\hline No. & Phase & Experimental & Control & Property \\
\hline NCT02273375 & III & MEDI4736 & Placebo & Adjuvant \\
NCT01407822 & II & Erlotinib & Gemcitabine/cisplatin & Neoadjuvant \\
NCT02511106 & III & AZD9291 & Placebo & Adjuvant \\
\hline
\end{tabular}

While adjuvant chemotherapy achieved a $4-5 \%$ improvement in 5-year OS, according to RADIANT and EVAN, at data cut-off, there were still more than $70 \%$ patients in TKI group were alive although final OS was immature $(5,7)$. So, adjuvant TKIs could bring survival benefits for resectable NSCLC.

As for the confusion about lung squamous carcinoma (LCS) with EGFR mutations, there are about $8.6 \%$ LCS harboring this variation (8). In speaking of relapse situation, after 24 months, the Kaplan-Meier curves for DFS survival began to converge, meeting by 36 months, with no apparent tail of non-recurrent patients in either treatment group by 48 months. One of the possible reasons might be the differences of baseline gene profile. Thus the genetic and immune landscape of patients enrolled needs to be further investigated. Furthermore due to the existence of minimal residual disease, growth-suppressed cells might be screened out and persist, ready to re-emerge on cessation of treatment. So the next critical step is to assess minimal residual disease to best define groups of patients, for example, detection of ctDNA (9).

At present, the development of targeted and immune therapy has refined the adjuvant treatment setting. An understanding of the immune landscape of tumors, including immune-evasion strategies, has led to breakthrough therapeutic advances. Immunotherapy has constantly burst its bounds and moved forward to early stage NSCLC. Nowadays there are nearly 100 ongoing trials focused on the adjuvant treatment around the world and we also participate in partial trials (Table 1). Since the premise of precision medicine is to select patients accurately, we sincerely hope the future adjuvant trials should figure out groups of patients who could benefit most from tyrosine kinase inhibitors or immunotherapy.

\section{Acknowledgements}

None.

\section{Footnote}

Conflicts of Interest: The authors have no conflicts of interest to declare.

\section{References}

1. Zhong WZ, Wang Q, Mao WM, et al. Gefitinib versus vinorelbine plus cisplatin as adjuvant treatment for stage II-IIIA (N1-N2) EGFR-mutant NSCLC (ADJUVANT/ CTONG1104): a randomised, open-label, phase 3 study. Lancet Oncol 2018;19:139-48.

2. Hirsch FR, Scagliotti GV, Mulshine JL, et al. Lung cancer: current therapies and new targeted treatments. Lancet 2017;389:299-311.

3. Pignon JP, Tribodet H, Scagliotti GV, et al. Lung adjuvant cisplatin evaluation: a pooled analysis by the LACE Collaborative Group. J Clin Oncol 2008;26:3552-9

4. Goss GD, O'Callaghan C, Lorimer I, et al. Gefitinib versus placebo in completely resected non-small-cell lung cancer: results of the NCIC CTG BR19 study. J Clin Oncol 2013;31:3320-6.

5. Kelly K, Altorki NK, Eberhardt WE, et al. Adjuvant Erlotinib Versus Placebo in Patients With Stage IBIIIA Non-Small-Cell Lung Cancer (RADIANT): A Randomized, Double-Blind, Phase III Trial. J Clin Oncol 2015;33:4007-14.

6. Mauguen A, Pignon JP, Burdett S, et al. Surrogate endpoints for overall survival in chemotherapy and radiotherapy trials in operable and locally advanced lung cancer: a re-analysis of meta-analyses of individual patients' data. Lancet Oncol 2013;14:619-26.

7. Yue D, Xu S, Wang Q, et al. Erlotinib versus vinorelbine plus cisplatin as adjuvant therapy in Chinese patients with stage IIIA EGFR mutation-positive non-small-cell lung cancer (EVAN): a randomised, open-label, phase 2 trial. Lancet Respir Med 2018. [Epub ahead of print].

8. Wu M, Pan X, Xu Y, et al. Methodological comparison 
of the allele refractory mutation system and direct sequencing for detecting EGFR mutations in NSCLC, and the association of EGFR mutations with patient characteristics. Oncol Lett 2018;16:1087-94.

9. Ng TL, Camidge DR. Lung cancer's real adjuvant EGFR targeted therapy questions. Lancet Oncol 2018;19:15-7.

Cite this article as: Liu SY, Wu YL. Adjuvant TKIs: still an optimal choice. J Thorac Dis 2018;10(12):E812-E814. doi: 10.21037/jtd.2018.11.67 